\title{
Wie gefährdet sind jugendliche Gothics?
}

Fragestellung: Ziel der vorliegenden Studie war es herauszufinden, ob die Identifikation mit der Gothic-Subkultur mit Depression oder selbstverletzendem Verhalten assoziiert ist.

Hintergrund: Frühere Studien zeigten einen engen Zusammenhang zwischen der Gothic-Subkultur sowie selbstverletzendem Verhalten und Suizidversuchen, wobei der Grund unklar blieb.

Patienten und Methodik: Für die Längsschnittstudie wurden Daten einer britischen Geburtskohorte, die 14.541 Schwangere mit Geburtstermin zwischen April 1991 und Dezember 1992 einschloss, verwendet. Ab sieben Jahren wurden die Kinder zu jährlichen Untersuchungen eingeladen. Mit 15 Jahren wurde das Ausmaß der Identifikation mit der Gothic-Subkultur erhoben und depressive Stimmungslage sowie selbstverletzendes Verhalten mittels standardisiertem Fragebogen gemessen. Mit 18 Jahren wurden Symptome einer

O'Connor RC, Portzky G. The association between goth subculture identifi cation, depression, and self-harm. www.lancet. com/psychiatry; 2015. http://dx doi.org/10.1016/ S2215

0366(15)00211-4 Depression sowie selbstverletzendes Verhalten mittels Clinical Interview Schedule - Revised (CIS-R) erfragt. Zudem wurden der elterliche Sozialstatus, mütterliche Depression, Konflikte mit Gleichaltrigen, Lebensereig- nisse, Internalisierung und Externalisierung von Problemen sowie die Selbsteinschätzung der Teilnehmer erhoben.

Ergebnisse: Von 5.357 Teilnehmern, zu denen Daten zur Identifikation mit der Gothic-Subkultur vorlagen, waren bei 3.694 Personen auch Daten zu Depression und Selbstverletzung verfügbar. Jugendliche, die sich nicht mit dieser Subkultur identifizierten, litten zu 6\% an Depressionen und zeigten zu $10 \%$ selbstverletzendes Verhalten. Im Gegensatz dazu erfüllten $18 \%$ derjenigen, die sich sehr mit der Gothic-Subkultur identifizierten, die Kriterien einer Depression; 37 \% dieser Gruppe berichteten von selbstverletzendem Verhalten. Es bestand eine DosisWirkungs-Beziehung zwischen dem Ausmaß der Selbsteinschätzung als Gothic mit Depression und selbstverletzendem Verhalten. Jene, die sich teilweise mit der Subkultur identifizierten, hatten ein 1,6-fach erhöhtes Risiko für eine Depression, jene, die sich sehr identifizierten, ein über dreifach erhöhtes Risiko. Ähnliche Ergebnisse zeigten sich für selbstverletzendes Verhalten.

Schlussfolgerungen: Die Ergebnisse weisen zwar auf ein erhöhtes Risiko für Depression und selbstverletzendes Verhalten bei Jugendlichen hin, die sich mit der Gothic-Subkultur identifizieren, die Autoren betonen jedoch, dass diese Beobachtung keinen Schluss auf die verursachenden Faktoren zulasse und empfehlen Präventionsmaßnahmen innerhalb jener Gruppe.

\section{Eine vulnerable Gruppe, aber keine Ursache-Wirkungs-Beziehung}

Zwischen dem Ausmaß der Identifikation mit der Gothic-Subkultur und dem Auftreten von Depressionen und selbstverletzendem Verhalten scheint es bei Jugendlichen eine Art DosisWirkungs-Beziehung zu geben. Dieser Zusammenhang erwies sich als robust selbst bei Berücksichtigung von potenziellen Störvariablen wie vorangegangenen depressiven Symptomen und Selbstverletzung, Persönlichkeitsfaktoren, Mobbing, Verhaltensproblemen, mütterlicher Depression oder Körperbild.

Es handelt sich hier um eine Studie der Evidenzstufe III, das heißt eine methodisch hochwertige Studie ohne Randomisierung. Die Studie verfügt über mehrere Stärken: Dazu zählen die große Teilnehmerzahl, das prospektive Studiendesign und die Fülle an verfügbaren Informationen über mögliche Störvariablen. Dennoch besteht die Möglichkeit für Confounding, wie auch die Studienautoren einräumen. So besteht die Möglichkeit, dass gerade jene Jugendliche, die anfällig für Depressionen sind, sich eher zu Subkulturen hingezogen fühlen, die bekanntermaßen einen Platz für marginalisierte Personen bieten. Des Weiteren sollte die nicht geringe Lost-to-follow-up-Rate beachtet werden; bemerkenswerterweise kamen diejenigen Jugendlichen, die alle vorgesehenen Untersuchungen absolvierten, aus besser gestellten Elternhäusern als diejenigen mit unvollständigen Daten. Eine mögliche Erklärung für den Zusammenhang stellt das Prinzip der sozialen Ansteckung dar. Als alternatives Erklärungsmodell bietet sich ein Attraktionsmodell an, das besagt, dass das Ausmaß, in dem Jugendliche sich zu jener Subkultur hingezogen fühlen, widerspiegelt, inwieweit sich Jugendliche von der Gesellschaft an den Rand gedrängt oder stigmatisiert fühlen.

Zusammenfassend lässt sich feststellen, dass es einen deutlichen Zusammenhang zwischen der Gothic-Subkultur und Depressionen oder selbstverletzendem Verhalten bei Jugendlichen gibt und dass diese eine besonders vulnerable Gruppe darstellen, dies jedoch nicht zur Annahme eines Ursache-Wirkungs-Prinzips verführen sollte.

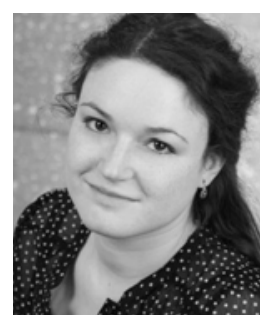

Dr. med. univ. Jessica Mattivi, Mainz

Klinik für Psychiatrie und Psychotherapie, Universitätsmedizin Mainz E-Mail: jessica.mattivi@unimedizin-mainz.de 